\title{
Clemens Knobloch
}

\section{Sprachwissenschaft}

\section{Das Zitat; Ziel der Untersuchung: eine Art Forschungsstand ${ }^{1}$}

„Die Sprachbetrachtung der Gegenwart geht ,aufs Ganze“." Das ist ein Zitat von 1934. Von wem es stammt, verrate ich einstweilen nicht. Beinahe jeder Sprachwissenschaftler könnte es geschrieben haben, beinahe jeder hätte es vermutlich unterscbreiben können. Der Satz geht folgendermaßen weiter: „Man kann zwar vom Ganzen zum Einzelnen gelangen, aber nicht umgekehrt vom Einzelnen zum Ganzen." Auch das dürfte eine in allen Lagern resonanzfähige Formulierung gewesen sein.

Eine gleichermaßen emphatische und programmatische Vorstellung vom „Ganzen" der Sprache, das theoretisch erfaßt und erklärt werden müsse, ist gewissermaßen der kleinste gemeinsame Nenner der sprachwissenschaftlich Tätigen in den 20 er Jahren. Es ist unbestimmtes Programm- und Fahnenwort einerseits, markiert die Frontstellung gegenüber allen Spielarten des einzelheitlichen "Positivismus“ andererseits.

Im Werk Bühlers, der wie kein zweiter geschult war an den Ganzheitslehren der Zeit (Gestaltpsychologie, Strukturalismus, Kultursoziologie), begegnet das "Ganze" häufig im Titel der Publikationen (z. B. 1931/32). An Saussures "Cours" wird bemängelt, daß er die Sphäre der Rede (parole), der Kommunikation, die Realität des Sprechereignisses einfach stehen läßt wie eine „unklärbare Mutter-

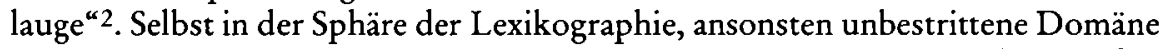
des "einzelnen" Lemmas und des sprichwörtlich positivistischen Alphabets, finden wir, begründet durch Ipsen, Trier, Porzig, den Feldgedanken. Dornseiffs ho-

1 Für zahlreiche wertvolle Hinweise insbesondere zu den romanistischen Akteuren des folgenden Textes danke ich Frank-Rutger Hausmann.

2 "Die Sprachforschung darf ebensowenig wie irgendeine andere Wissenschaft von dem, was sie als Ausgangsgegenstand einmal aufgenommen hat, prinzipiell irgend etwas wie eine unklärbare Mutterlauge sozusagen stehen lassen." So heißt es in der "Axiomatik" (Bübler 1933:38), Bühlers Art, ,aufs Ganze' zu gehen, bleibt darin auch aus heutiger Sicht immer „fachlich“, als er an der allseitigen Indexikalität der Sprache als ihrer Funktion interessiert ist, nicht an den fallweise indizierten weltanschaulichen "Inhalten“, die eben darum rasch wechseln. 
listisch und „synonymisch“ konzipierter „Wortschatz nach Sachgruppen“ erscheint ab 1933.

Das "Ganze " begegnet freilich in zahlreichen und rasch wechselnden Gestalten. Es begegnet in der kulturalistischen Philologie und Etymologie, in der „Wörter und Sachen"-Schule, es begegnet als "System“, als "Gestalt", als „Feld“, als "Sprechereignis", als „Geist", als "Sprachgemeinschaft" - und schließlich auch als „Volk“, als „Sprachvolk“ nämlich.

Nicht weniger auffällig als der Hang zum "Ganzen ${ }^{\circ}$ ist in der Sprachwissenschaft der 20er Jahre der Wille zum programmatischen Neubeginn, präsent in unzähligen Publikationen, welche den gegenwärtigen „Stand“ bilanzieren und programmatisch künftige Forschungslinien vorzeichnen. Sehr gefragt sind offenbar Axiomatisierungsversuche, die neuen Halt versprechen im Strudel der Krisenpolyphonie. Vor dem Hintergrund dieser „programmatischen“ Publikationen will ich der Frage nachgehen, ob es tatsächlich NS-spezifische Weisen gegeben hat, in sprachwissenschaftlichen Dingen, aufs Ganze' zu gehen.

\section{Skizze der Ausgangslage um 1930; Verabschiedung der gängigen Mythen vom „Sonderweg“ der deutschen Sprachwissenschaft}

Hier, bei den zirkulierenden „Mythen“ über die deutsche Sonderentwicklung in der Sprachwissenschaft, fasse ich mich kurz und verweise auf die einschlägige Literatur:

Mythos 1: die deutsche Sprachwissenschaft habe sich an Saussure und dem Strukturalismus vorbei entwickelt. Das ist grundfalsch. Vielmehr gilt ohne Einschränkung, was Bühler (1934), zweifellos der beste Beobachter der zeitgenössischen Sprachtheorie, notiert: daß seit dem Erscheinen des "Cours" kein Sachkundiger mehr an der Reflexion von Langue und Parole, Synchronie und Diachronie vorbeikommt. In Weisgerbers handgeschriebener Habilitationschrift von 1925 ("Sprache als gesellschaftliche Erkenntnisform“) ist Saussure der meist zitierte Autor ${ }^{3}$. Trier (1931) beruft sich ständig auf den "Cours“, Gunther Ipsen (1930), eine Schlüsselfigur der Reorganisation der deutschen Sprachwissenschaft in den 20er $\mathrm{Jahren}^{4}$, nennt ihn unter den wichtigen Neuerungen etc. Und auch über die gesamte NS-Zeit hinweg sind strukturalistische Positionen präsentabel und präsent.

${ }^{3}$ Hierzu vgl. man besonders Eblers (2000). Auch in den frühen Publikationen Weisgerbers, so insbesondere in dem Handbuchartikel "Sprache“, den dieser zu Vierkandts „Handwörterbuch der Soziologie" beisteuerte (Weisgerber 1931), ist überdeutlich, daß Weisgerber die psychologistisch-soziologistische Ambivalenz des "Cours" zur soziologistischen Seite hin auflöst. 4 Vgl. Ipsen (1930); Gunther Ipsen hat sich später stärker in der Soziologie engagiert. Er gilt als Mitbegründer der Leipziger Soziologenschule und ist auch in der Geschichtswissenschaft der NS-Zeit kein Unbekannter, weil er an der Konzipierung der NS-Raumpolitik im Osten beteiligt war. Als Wissenschaftler und Wissenschaftspolitiker hätte er definitiv eine Monographie verdient. 
Vielmehr ist es auch gar nicht zu übersehen, daß der deutsche Hang, aufs Ganze' zu gehen, sich durchaus auch auf Saussure berufen konnte und sich auch auf ihn berufen hat.

Mythos 2: Die deutsche Sprachwissenschaft habe sich im Nationalsozialismus international isoliert. Tatsächlich ist die relative Isolierung der deutschen Sprachwissenschaft bereits früher eingetreten. Sie geht zurück auf die Abgrenzung der europäischen und internationalen Sprachwissenschaften gegen die bis dahin übermächtige deutsche Tradition nach dem Ersten Weltkrieg, die mit neuem antideutschem Selbstbewußtsein und vornehmlich im Zeichen des Strukturalismus erfolgte (Maas 1988:263f.). Überdeutlich ist bereits in den 20er Jahren und erst recht im Nationalsozialismus der deutsche Anspruch, die alte Führungsrolle in der Sprachwissenschaft wiederherzustellen, was naturgemäß nicht durch einfachen Anschluß an die internationale Entwicklung geschehen konnte, sondern deren Überbietung einschloß. Auch daher der emphatische Hang zum „Ganzen“. Es ist leicht nachweisbar, daß in der Sprachwissenschaft wie in den geisteswissenschaftlichen Nachbarfächern Einsicht in die „Ganzheitlichkeit“ geistiger Phänomene als spezifisch deutsche Leistung reklamiert und somit national übercodiert wurde (Stempel 1978, Scheerer 1991) 5 .

Mythos 3: Die Sprachwissenschaft im Nationalsozialismus sei durchweg wertlos, ideologisch korrumpiert, gleichgeschaltet und kein legitimer Gegenstand der Fachgeschichte. Dagegen ist zu halten, daß es in der Entwicklung der Sprachwissenschaft nach 1933 keinen Bruch gegeben hat. Die Programmschriften und Axiomatiken der gewöhnlich für "Nationalsozialismus“ geltenden Richtungen sind vor 1933 erschienen. Keine relevante Strömung der Weimarer Krisenpolyphonie ist nach 33 untergegangen, und keine ist neu dazugekommen. Auch die rassistisch argumentierenden Strömungen debütieren vor 33. Vielmehr wirkt die durchgesetzte NS-Ideologie als Kraftfeld und zusätzlicher Selektionsfaktor (man könnte auch sagen: als Differenzierungshilfe) für die „Auslese“ der akademisch institutionell stärksten Richtungen. Sprechen kann man jedoch nicht von einer Gleichschaltung des Faches, sondern bestenfalls von seiner Selbstgleichschaltung ${ }^{6}$.

5 Es sei daran erinnert, daß schon Ringer (1983:344 ff. und öfter) auf die "Bewegung zur Synthese" und auf die rhetorische "Technik des höheren Dritten“ im Krisendiskurs der Zeit aufmerksam gemacht hat. Untersuchenswert wäre noch der Umstand, daß es im Ganzheitsrausch des Nationalsozialismus dann alsbald auch die Rolle des Ernüchterers gibt. Willy Hellpach, dessen dreistufige Volkslehre (Hellpach 1938) als klassische Durchführung der NS-Volkstheorie gelten kann (1. Stufe: „Volk“ als Naturtatsache, Blut; 2. Stufe: „Volk“ als geistige Gestalt, Sprache, Tracht, Werkzeug etc.; 3. Stufe: „Volk“ als Willensschöpfung, Bund, SS etc.), veröffentlicht 1937 in der Zeitschrift „Industrielle Psychotechnik“ (der Ort ist so interessant wie die Zeit) eine ironisch-begriffskritische Analyse der "Ganzheitsmystik“. Der Text ist intelligent und interessant, er nimmt eine Art Hermann-Lübbe-Position zum Ganzheitsdiskurs an, eine Art affirmative Relativierung. "Wer nichts als Ganzheitsvisionen hat, der bleibt ein Träumer, ein Phantast, ein Schwärmer “, heißt es da (Hellpach 1937:133), und gewarnt wird vor den „Gefahren der Einzelheitsvernachlässigung“ (1937:138).

6 Und was die "geduldete Mehrstimmigkeit" des Faches im Nationalsozialismus betrifft, so verdient es einmal bemerkt zu werden, daß die bundesdeutsche Nachkriegssprachwissen- 
Mythos 4: Die Politisierung des Faches sei eine Funktion des Nationalsozialismus und habe „entwissenschaftlichend“ gewirkt. Tatsächlich ist bekannt, daß der Ruf, Sprachwissenschaft müsse zu den praktischen Fragen des Tages, der Schule, der Politik, der Kommunikation, der Völkerbeziehungen, der Propaganda Sachdienliches beitragen, bereits mit der „antipositivistischen “ Kritik an den junggrammatischen "Lautschiebern" (Vossler) aufgekommen ist. Die völkische Jugendbewegung, die Verbindung zu den Grenz- und Auslandsdeutschen Aktivisten kam in der Zeit des Ersten Weltkrieges radikalisierend hinzu. Programmschrift und Geburtsurkunde der Sprachinhaltsforschung ist die im Jahre 1917 von Georg Schmidt-Rohr (damals, bis ca. 1930, noch einfach Georg Schmidt) ${ }^{7}$ publizierte Tat-Flugschrift mit dem bezeichnenden Titel: „Unsere Muttersprache als Waffe und Werkzeug des deutschen Gedankens". Bei ihrem Machtantritt fanden die Nazis eine durchweg politisierte Sprachwissenschaft bereits vor. Was das Personal betrifft, so reicht das Spektrum vom politischen Aktivisten, dem die "Wissenschaft" ebenfalls "Waffe und Werkzeug" seiner völkischen Sendung ist, über den sozial intelligenten Karrieristen und den modernen Politikberater bis hin zum peniblen Philologen, der seine strenge methodologische Haltung mühelos mit "völkischer Verantwortung", banalem Alltagsnationalismus und „deutscher Wissenschaft" zur Deckung bringt ${ }^{8}$.

Unklar und von einer soziologisch nicht reflektierten Wissenschaftsvorstellung ausgehend ist weiterhin die Ansicht, ein solcher praktisch politischer Kontext gehe notwendig zulasten der "Wissenschaftlichkeit“. Vielmehr zeigt auch hier der Extremfall der manifesten Politisierung nur deutlicher, was auch im Normalbetrieb vor sich geht. Der Zwang, verwertbare Ergebnisse zu liefern, diszipliniert auch dann ein Fach, wenn er direkt politisch motiviert ist. Er baut das Fach in kürzere Kontrollschleifen ein, erzwingt objektivere Methodiken und kontrolliert wirksam ideologischen Überschwang9 ${ }^{9}$. So gilt, daß die deutsche Sprachsoziologie,

schaft mit ihrer Weisgerber-Monokultur sehr viel grauer, monotoner und fachlich unproduktiver gewesen ist.

$7 \mathrm{Zu}$ Schmidt-Robr vergleiche man vor allem die zahlreichen Publikationen von Gerd Simon (1979, 1985, 1986, 1989 und öfter). Vgl. auch Knobloch (i. V.), jetzt auch einige Bemerkungen in Hutton (1999).

${ }^{8}$ Zweifellos hat diese „Politisierung“ in Deutschland eine spezifische, wohl ebenfalls auf den Ersten Weltkrieg zurückführbare Form angenommen, als Phänomen ist sie aber auch anderswo zu beobachten, wie im Zeitalter der nationalen und sprachlichen Minderheiten nicht anders zu erwarten. Weisgerber (1934:204) zitiert A. Meillet, den er häufig als seinen französischen Antipoden führt (und als den „Führer der französischen Sprachforschung“ bezeichnet) mit dem Satz, die Aufgabe der Wissenden sei, diejenigen aufzuklären, die handeln müssen.

9 Hier wäre zuerst von den Fächern zu lernen, die ihre Professionalisierung dem Krieg oder sonstiger politischer Indienstnahme verdanken, so z.B. der Psychologie und der Soziologie. Für die „Professionalisierung“ der deutschen Psychologie im Nationalsozialismus vgl. Geuter (1984), Ash \& Geuter (1985), für die Soziologie Klingemann (1996). Bekannt ist auch in diesem Zusammenhang, daß Wissenschaftskarrieren zwar in der Konsolidierungsphase des Nationalsozialismus mit ideologischer Kollusion begründet werden konnten, in der Kriegsphase aber nur noch mit verwertbaren Ergebnissen (Maas 1996). Das gilt selbst für ein so ab- 
die Varietätenlinguistik, die Sprachkontaktforschung, die Bilingualismusforschung, die Lernergrammatik für $\mathrm{DaF}$ ihren Anfang in derartigen politisierten Kontexten genommen haben. Wo steht geschrieben, daß „reiner" Erkenntnisdrang besser sehen lehrt als profaner Verwertungsdruck? Das führt uns bruchlos zum

Mythos 5: Und der beinhaltet die These von der ideologischen Korruption des Faches im Nationalsozialismus, die eigentlich erst in der durchgreifenden Ausmistung durch die Studentenbewegung ihr Ende gefunden habe. Hier ist zu bemerken, daß nichts die realistische Einschätzung der Sprachwissenschaft im Nationalsozialismus so sehr behindert wie die optische Täuschung, die durch den verspäteten Anschluß der deutschen Linguistik an die internationale strukturalistische Entwicklung in den 60er Jahren installiert worden ist ${ }^{10}$. Hier ist ein enormer semantischer Entwertungsdruck aufgebaut worden, der es nahelegte, die bis dahin vorherrschenden Ansichten pauschal als NS-infiziert zu codieren, was ihren raschen semantischen Verschleiß zu fördern versprach und ihre kritische Prüfung verhinderte. Mit den Karriereinteressen derer, die der Weisgerberei ihr wohlverdientes Ende bereiten wollten, war dieser sprunghafte semantische Verschleiß untrennbar verbunden. Die Weisgerberei war einfach nicht mehr resonanzfähig, weshalb man ihre Axiome auch durchaus nicht mehr zu prüfen brauchte ${ }^{11}$. Man darf jedoch nicht vergessen, daß es keine "fachlichen" Gründe waren, welche die bis dato allmächtige Sprachinhaltsforschung rasch kollabieren ließen, sondern die Koinzidenz von Resonanzverlust und den Karriereinteressen der nachrückenden Linguistengeneration.

Mythos 6: Die Sprachinhaltsforschung habe tapfer das Banner des "geistigen“ Prinzips der Muttersprache gegen die Rassenbarbarei der Nazis aufgepflanzt und

gelegenes Fach wie die Sprachwissenschaft, wo man am Schicksal Schmidt-Rohrs studieren kann, daß Nützlichkeitsversprechungen rasch eingelöst werden müssen, wenn sie nicht zum Karriereknick führen sollen (vgl. Simon 1986, 1989 über Schmidt-Rohr). Ein Mann wie Heinz Kloss war da wesentlich effizienter. Er lieferte bienenfleißig und vollkommen realistisch die Daten, Zahlen und Fakten, welche der Krieg benötigte, so z. B. über die Mobilisierbarkeit der Deutsch-Amerikaner gegen einen Eintritt der USA in den Krieg (Kloss 1937 zuerst, hierzu Maas 1996:73), über Zahl und Einfluß der jüdischen Organisationen in den USA (Kloss 1944), über die sprachlichen und ethnischen Minderheiten in Frankreich (Kloss 1935) u.v.a.m.

10 Bedenkenswert ist hier nicht zuletzt, daß für die deutsche Sprachwissenschaft fast ein halbes Jahrhundert Denkentwicklung ausgespart geblieben ist. Eine genuine Rezeptionslücke besteht zwar nicht für Saussure, wohl aber für große Sprachforscher wie: Jespersen, Benveniste, Kurylowicz, Meillet, Bally, Tesnière, Jakobson, Bloomfield, Hockett, Pike und einige andere mehr.

11 Ich bin mir darüber im klaren, daß diese historische Relativierung des plötzlichen Endes der Weisgerberei diejenigen auf den Plan rufen wird, die schon immer behauptet haben, hier sei fachgeschichtliches Unrecht geschehen. Es versteht sich jedoch, daß ich durchaus nicht dieser Meinung bin, im Gegenteil. Fachgeschichtliches Unrecht gibt es nicht, jedenfalls so nicht. Der plötzliche Zusammenbruch eines außerfachlichen Resonanzraumes bestätigt vielmehr in seinen weitreichenden Wirkungen, daß es der Sprachinhaltsforschung nicht gelungen war, eine halbwegs resistente Fachtradition aufzubauen. Diese Diagnose gilt freilich auch für die Linguistik bis heute. 
sei darin trotz Anfeindungen keinen Zoll gewichen. Das ist der Stoff, aus dem die Persilscheine nach 45 gefertigt wurden. Eine Tatsache ist, daß die völkische Muttersprach-Rechte zur "ideologischen Koalition“ der Nazis gehörte und ihnen bis zum Schluß treu gedient hat (Hutton 1999:4). Wahr an dieser Gechichte ist hingegen, daß nachhaltig zurückgepfiffen wurde, wer sich in das Geschäft einzumischen suchte, das Partei und „Bewegung“ für sich vorbehalten glaubten: das der Definition und Gewichtung zentraler ideologischer Konzepte wie "Volk“, „Rasse“, „Arier“. Das mußte z.B. Schmidt-Rohr erfahren, dessen Tiraden gegen das Rasseprinzip im Volksdiskurs, zumal er sie nach der Machtübergabe 1933 erneut drucken ließ, zu einem ernsthaften Karriereknick führten (zu mehr freilich nicht). Der endete erst, als Schmidt-Rohr, der fortfuhr, Parteidienststellen mit sprachpolitischen Programmen zu beschäftigen, 1939 mit dem Segen höchster Parteiideologen widerrief ${ }^{12}$. Insofern bedeutet die Machtübergabe an die Nazis 1933 tatsächlich eine relative "Schließung" des zentralen ideologischen Diskurses der völkischen Rechten. Und davon war die Muttersprach-Fraktion insofern betroffen, als sie Teil dieses völkischen Diskurses gewesen (und geblieben) ist ${ }^{13}$.

\section{Kraftzentren der Fachentwicklung zwischen 33 und 45; Was bewegt die „Sprachwissenschaft“?}

Daß es "die" Sprachwissenschaft als halbwegs geschlossene akademische Konfiguration im Nationalsozialismus nicht gab, ist bekannt (vgl. die Diskussion in Maas 1988 und 1996). Auch Fachgrenzen stellen sich im Rückblick anders dar als für die Zeitgenossen selbst. So wurde in den Rezensionsorganen des ersten Jahrhundertdrittels - um nur ein Beispiel zu nennen - vielfach unter der Rubrik „Sprachpsychologie“ geführt, was wir heute der Allgemeinen Sprachwissenschaft oder Sprachtheorie zurechnen würden ${ }^{14}$. Auch gegen diesen terminologisierten Monopolanspruch der Psychologie in sprachtheoretischen Fragen richtet sich der „antipsychologische" Groll der Neuerer.

Mit Maas $(1988,1996)$ entscheide ich mich retrospektiv für einen „weiten“ Begriff von Sprachwissenschaft, der sowohl unter den „Philologen“ die vornehmlich

12 Hierzu vgl. man Schmidt-Rohr (1939a und 1939b). Der "Widerruf“ wurde eingeleitet von Prof. Dr. Groß, Leiter des Rassenpolitischen Amtes der NSDAP. Eine detaillierte Rekonstruktion des Konfliktes zwischen Schmidt-Rohr und der NSDAP gibt Simon (1986).

13 Das zentrale Thema dieses Diskurses war die Frage nach den "volksbildenden“ Kräften (Raum, Sprache, Kultur, Rasse etc.), nach ihrer Gewichtung und Hierarchisierung. Versorgt wurde er aus dem Umkreis der Strömungen, die heute vielfach unter der Chiffre der „Konservativen Revolution“ zusammengefaßt werden. Ein zentraler, repräsentativer und wirkungsmächtiger Text ist Boebm (1932). Zu Boehm vgl. Petzold (1978).

${ }^{14}$ Das hat natürlich seinen fachhistorischen Grund darin, daß im 19. Jahrhundert die Psychologie mit einigem Erfolg ihre Zuständigkeit für die Fragen der Sprache erklärt hatte; vgl. Knobloch (1988). In der Generation von Wilhelm Wundt und Hermann Paul war diese Zuständigkeit weitgehend anerkannt. 
an Sprache Interessierten umfaßt als auch die sprachpraktisch und sprachpolitisch Tätigen im außerakademischen Bereich, ebenso auch den Komplex Sprachpflege, der (zusammen mit dem Allgemeinen Deutschen Sprachverein) freilich bereits relativ gut erforscht ist ${ }^{15}$. Ich will im folgenden Abschnitt einige "Kraftzentren der Sprachwissenschaftsentwicklung im Nationalsozialismus umreißen. Darunter will ich umgrenzbare thematische, institutionelle und semantische Komplexe mit „Ressourcen“-Charakter (Ash 1995a, 1995b) verstanden wissen. Eigentümlich ist diesen Kraftzentren, daß die Beziebbarkeit der fachlichen Arbeit auf einen oder mehrere dieser Komplexe verbesserte Resonanz verspricht. Der erste dieser Komplexe ist:

a. Das (durchweg als sprachlich und „volklich“ bedroht codierte) Grenz- und Auslandsdeutschtum. Hier gibt es eine emphatische Tradition der Muttersprachund Volksgemeinschaft, für die als Organisation die Gruppen der bündischen Jugend im und nach dem Ersten Weltkrieg stehen. Deren wichtigster Vertreter ist Georg Schmidt-Rohr. Seine (o.g.) Tat-Flugschrift von 1917 kann als erstes Manifest und Geburtsurkunde der ganze Richtung gelten. Auch Weisgerbers lebenslange Obsession mit "Sprachkampf“ und „Sprachgrenze“ führt (selbst biographisch) zurück auf diesen Hintergrund (Weisgerber stammt aus Lothringen). Das Netz der Grenz- und Auslandsdeutschen Hilfsorganisationen kann hier nicht rekonstruiert werden. Ich nenne nur das Deutsche Ausland-Institut (Stuttgart) und als wichtigen Sprachwissenschaftler daselbst Heinz Kloss.

Die (vermeintlichen) Anliegen der Grenz- und Auslandsdeutschen versprachen beträchtliche Resonanz schon in der Weimarer Republik, erst recht im Nationalsozialismus. Für die völkische Sprachwissenschaft bildeten sie eine wichtige Schnittstelle zwischen Theorie und Praxis. Als kleine, von „volksfremden" Mächten umgebene Sprachgemeinschaften erlaubten sie das Studium der sprachlichen Vergesellschaftung (ebenso wie des Sprach- und Volkstumsverlustes!) unter feindlichen Bedingungen - und leisteten automatisch einen Beitrag zu hoch „nationalen" Anliegen ${ }^{16}$. Fast automatisch wurde das Grenz- und Auslandsdeutschtum

15 Vgl. hierzu Polenz (1967), Simon (1979, 1989), Bernsmeier (1983), Kämper-Jensen (1993). 16 Der Mythos vom sprachlichen und völkischen „Grenzkampf“" war so zugkräftig, daß auch die rassistische Fraktion ihn, nur leicht mit Blut unterfüttert, nicht entbehren zu können glaubte (vgl. Glässer 1939:22f. und öfter). Nur wo die rassischen Kräfte erlahmt sind, unterliegt auch die Sprache etc. Anschluß bietet der Topos jedoch auch für höchst fachliche Fragen von Sprachmischung, Sprachkontakt, Entlehnung, Substrat- und Superstratbildung etc., die alle ebenfalls auf Sprach- und Grenzkämpfe „zurückgeführt“ werden können. Grundsätzlich lassen sich alle Wirkungen des Sprachkontaktes auch als Wirkungen von "Sprachkämpfen" codieren. Und allfällige Befunde, wonach die "Nationalsprachen “ höchst kontingente Mischungen mit großen Anteilen von Entlehnung und Lehnübersetzung bilden (vgl. Maas 1996:61 f.), lassen sich gleichermaßen bereitwillig auf „Völkermischungen“ wie auf „Völkerkämpfe " beziehen. Versteht sich, daß der NS-Diskurs die kämpferische Lesart prämierte und die "nationalliberale" schmähte. Neben Hugo Schuchardt, den Maas (1996) in diesem Zusammenhang nennt, ist vor allem an den skeptisch-mystischen Sprachkritiker (und Außenseiter) Fritz Mauthner zu erinnern, dem es allzeit ein Vergnügen war, den Misch- und Lehncharakter der vermeintlich reinen Nationalsprachen detailreich aufzuzeigen. NS-Kollusionsartisten wie Edgar Glässer (1939:33ff.) haben ihn dafür immerhin beschimpft, statt ihn nur 
Symbol und Barometer für das geschlagene, eingekreiste und gedemütigte „Gesamtvolk" - und gleichsam zum Referenzsystem für das, was die "volksbildende Kraft der Muttersprache" hieß. Auch setzten beträchtliche Teile der nationalen Intelligenz nach dem verlorenen Weltkrieg auf eine Art von geistig-kulturellem Imperialismus, auf einen Modus der Expansion, welcher sich auf die „Weltgeltung" der deutschen Sprache und Kultur zu stützen gedachte.

b. Der Komplex der deutschen Sprach- und Kulturpropaganda im Ausland. Personell und institutionell steht er in engem Zusammenhang mit (a). Institutionell am wichtigsten dürfte die Deutsche Akademie (DA München) gewesen sein ${ }^{17}$. An der arbeitet bis 1936/37 als wichtigster Sprachwissenschaftler Franz Thierfelder. Die DA hat Schmidt-Rohrs Hauptwerk (Schmidt-Rohr 1932, 1933) herausgegeben und seine Verbreitung gefördert. Kloss und Thierfelder haben das Buch in emphatischen Rezensionen begrüßt (vgl. Hutton 1999: 298ff.). Weisgerbers frühes Hauptwerk „Muttersprache und Geistesbildung" von 1929 kann durchaus als programmatisch akademische Ausgestaltung dieser Richtung gelesen werden.

Als semantische Ressource dürfte für diesen Komplex wichtig sein, daß der expansive Sprachnationalismus in Deutschland auch eine massendemokratische Mutations- und Schwundform des bildungsbürgerlichen Kultur- und Geisteselitismus darstellt. An den kann er auch insofern unmittelbar anschließen, als ja die deutsche Muttersprache der Schlïssel zur "deutschen Bildung und Kultur" ist ${ }^{18}$. Weisgerber (1933/34), zweifellos der begabteste Wissenschaftspolitiker seiner Fachgeneration, baut den Themen- und Entwicklungsplan der Sprachwissenschaft so, daß ihr die gatekeeper-Position in allen höheren Gemeinschaftsangelegenheiten automatisch zufällt, kraft der Tatsache, daß die eben alle muttersprachlich vermittelt sind.

zu ignorieren. Ein Zitat sei erlaubt, weil es den Ton Glässers exemplarisch vorführt: „Sucht er [Fritz Mauthner; $\mathrm{CK}$ ] doch die Eigenständigkeit und das nationale Gepräge der Sprachen immer wieder gleichsam zu entlarven als ein getarntes Volapük, das dem Wissenden das Freimaurerzeichen ahasverischer Allerwelts- und Nirgendwo-Beheimatung zu erkennen gebe. So schwelgte er in der Vorstellung von einem restlos vermischten Völkerbrei, von einer universalistischen Verkehrssoziologie, einem grenzenlosen Hin- und Herüber der Lehnübersetzungen..." usw. Glässer (1939) ist ein intelligentes Panoptikum der polit-rhetorischen Potentiale, die in den kurrenten sprachwissenschaftlichen Axiomatiken steckten. Schon darum verdiente der Text eine gründliche Analyse.

17 Zur Geschichte der Deutschen Akademie, insbesondere im Nationalsozialismus, vgl. Harvolk (1990), der auch die Streitereien rekonstruiert, die zum Ausscheiden Thierfelders geführt haben. Daß dieser Schwierigkeiten mit der (späteren) Gleichschaltung der DA gehabt habe, wie er nach 1945 behauptet, ist ein offenkundiges Märchen. Seine späteren Veröffentlichungen im Krieg sprechen eine andere Sprache (Thierfelder 1940, 1941). Laut Maas (1988:277) war Thierfelder nach seinem Ausscheiden bei der DA als sprachpolitischer Berater für das AA und für die SS tätig, nach dem Krieg dann für das Institut für Auslandsbeziebungen, Nachfolger des Auslandsinstituts in Stuttgart, und beim Goetheinstitut.

18 Interessant sind immer die äußersten Grenzen der Strapazierfähigkeit eines Interpretationsmusters. So formuliert Glässer (1939:3) als Ziel des neuphilologischen Unterrichts, die „Unübersetzbarkeit“ des sprachlichen Lebens einzusetzen und zu vermitteln. 
c. Der Komplex „Verwissenschaftlichung der Propaganda“. Hier ist der (u.a. von Weisgerber) reklamierte Charakter der Muttersprache als Trägerin der naturwüchsigen „Weltanschauung" einschlägig ${ }^{19}$. Es versteht sich, daß aus der (in Grenzen realistischen) Erkenntnis, daß Weltanschauungen sprachlich zirkulieren und sprachlich vermittelt werden, automatisch eine weitreichende Zuständigkeitserklärung der Sprachwissenschaft resultiert. Die kann in diversen Versionen vorgetragen werden, umweglos wie bei Eduard Hermann (1937) oder durchweg fachlich verklausuliert und bloß in der Allusion politisch wie bei Weisgerber (1933/34). Beide Techniken, das Fach in dieser Sache „in Stellung zu bringen“, müssen sehr viel genauer untersucht werden. Karrieren wie die von Manfred Pechau im Amt Rosenberg oder Franz Alfred Six als "Gegnerforscher" (Hachmeister 1998) sind auf diesen Komplex gegründet.

d. Die Sprachpolitik in den besetzten Ländern. Hier kann ich nicht ins Detail gehen. Am besten untersucht ist bislang die Sprachpolitik in den besetzten Niederlanden durch die Arbeiten von Christoph Sauer $(1989,1995)$ und die Sprachpolitik in der Bretagne durch Joachim Lerchenmüller (1997).

e. Der Komplex „Rassismus“. Hier handelt es sich um einen reinen Kollusionstopos. Der Rekurs auf „Rasse“ macht von allen positiven spachstrukturellen Vorgaben frei für jegliche programmatische Spekulation (Maas 1988:281), weshalb er für zwei Funktionen verwendbar wurde: für die Demonstration weltanschaulicher Übereinstimmung mit dem Nationalsozialismus (z.B. Lutz Mackensen, Hermann Ammann, Hermann Güntert und einige andere mehr) und für das (demonstrativ opportunistische) Freiräumen fachlicher Spielräume. Edgar Glässer (1939) ist der einzige Sprachwissenschaftler, der gleichzeitig offen rassistisch und auf der Höhe der sprachtheoretischen Diskussion schreibt. Seine „Einführung in die rassenkundliche Sprachforschung" ist der eigentliche Anti-Schmidt-Rohr und Anti-Weisgerber. Die rhetorische Makrostruktur des Textes ist freilich mehr als simpel. Überall da, wo die sprachnationalistische Fraktion "geistig“, „kulturell“, letztlich eben sprachlich ,aufs Ganze' geht (und dafür Struktur- oder Geschichtsdaten beibringen muß!), pocht Glässer auf "wuchshaft "-rassischen Urgund derselben: bei der Verständigung, bei den Lehnübersetzungen, bei den idg. Satzschemata etc. Es ist ja nicht eben schwer, alles, was hoch bewertet und geschätzt wird, günstigem Bluteinfluß zuzuschreiben und alles andere der allenthalben drohenden Mischung und Degeneration. All das wird von Glässer mit stupender Gelehrsamkeit und durchaus guter Detailkenntnis vorgetragen, bedenkt man, daß er zur Zeit der Abfassung 28 Jahre alt war. Krude tagespolitische Invektiven wechseln sprunghaft mit breit angelegten Theoriedebatten, letztere auf dem Sachstand der Vor-NS-Zeit (also mit Berücksichtigung von Saussure, Bühler, Meinong, Marty, Mauthner, Cassirer etc.). Für die primitiv-rassistische Auflösung wird dann am

19 Es würde durchaus einmal eine Untersuchung vertragen, wie die Sprachinhaltsforschung vorgeht, wenn sie den "geistigen " Charakter der Sprachen betont - "Geist" konnotiert ja Freiheit und Selbsttätigkeit - und gleichzeitig die Muttersprache als naturhaftes semantisches Gefängnis, als naturhaft-objektive Macht über den einzelnen, vorstellt. 
Ende regelmäßig Stegmann von Pritzwald zitiert, vor allem dessen anti-slawische Ausfälle $^{20}$. Der war offenbar Glässers Förderer und sprachsoziologischer Vertrauensmann des Amtes Rosenberg, was den (in jeder Hinsicht) gemeinen Karrierismus als Motiv nahelegt ${ }^{21}$. Daß Glässers Hauptgewährsmann in sprachtheoretischen Angelegenheiten ausgerechnet Karl Bühler war - Glässer gibt sogar eine eigene Version des Organonmodells, "gliedhaft“ bzw. „wuchshaft" umgeschminkt (beides seine Lieblingsadjektive [Glässer 1939:78f.]) - legt den Schluß nahe, daß der intelligente, ständig im Überbietungsgestus schreibende Jungtürke eine Strategie der symmetrischen Ausdehnung des diskursiven Raumes betrieb: Je mehr demonstrativer Rassismus auf der einen, desto mehr fachlicher Spielraum auf der anderen Seite 22 .

f. Der Komplex Sprachpflege und Purismus. Hier ist vor allem zu bemerken, daß der Nationalsozialismus die in ihn gesetzten Erwartungen puristischer Deutschlehrer und enthusiastischer Frakturfreunde nicht erfüllt hat. Letztere fühlten sich gar verraten, als der Führer 1940 die Verwendung der Antiqua in den offiziellen Dokumenten von Staat und Partei anordnete, namentlich im Hinblick auf die besetzten Gebiete, und den Bund für deutsche Schrift auflösen ließ (vgl. hierzu Hartmann 1998).

Obwohl natürlich puristisch-sprachpflegerische Rhetorik von vielen NS-Größen an der Öffentlichkeit praktiziert wurde - sie ist schließlich immer populär ist doch die interessantere Beobachtung die, daß mit derartigen Motiven allein auch im Nationalsozialismus keine akademische Karriere zu bestreiten war. Am weitesten dürfte es auf diesem Feld der Erlanger Rhetorik-Professor Ewald Geißler gebracht haben, Autor des Duden-Stilwörterbuchs, vorgesehen als Leiter des Sprachamtes der Deutschen Akademie (das dann nicht zustandekam; vgl. Simon 1989, 1990), Verfasser einer zweibändigen „Erziehung zur Hochsprache“, deren zweiter Band den Weg vom Weimarer Alltagsnationalismus zur markigen

20 Mein Exemplar von Glässer (1939) trägt die Widmung des Verfassers an Erich Rothacker, der das Buch sorgfältig mit Annotaten versehen hat, darunter auch die Bemerkung, daß Stegmann von Pritzwald ein slawischer Name sei. Mit der „Einführung in die rassenkundliche Sprachforschung “ hat Glässer sich in Heidelberg habilitiert. Nach dem Krieg war er in Mainz Direktor des Dolmetscherinstituts.

21 Namentlich gegen Weisgerber und Schmidt-Rohr holt Glässer mächtig aus: letzterer rechtfertige den "Völkerbrei“ mit seinem „wurzellosen Sprachbegriff“ und betreibe den „Einbau des Judentums in die Front der Nation“ (Glässer 1939:52).

22 Auch die höchst intellektualistische Saussure-Exegese der Kopenhagener Schule, mit ihrem Hang zur logizistischen Allgemeinen Grammatik der völkischen Schwärmerei maximal entgegengesetzt, versucht Glässer als „arteigen “ einzugemeinden (Hjelmslev, Brøndal), wohl weil sie in Skandinavien beheimatet ist. Die Argumentation ist bisweilen so beliebig, als ob sich Glässer darüber klar gewesen wäre, daß man mit Hilfe des Rasse-Musters in der Sprachwissenschaft alles erklären könne. Und das heißt eben: nichts. Es dürfte nicht leicht sein, einen anderen NS-Linguisten zu finden, der so souverän (und fast ironisch) mit den rhetorischen Resonanzpotentialen sprachwissenschaftlicher Theorie operiert wie Glässer. M. W. ist sein Büchlein unter diesem Gesichtspunkt noch nicht erschöpfend analysiert worden. 
NS-Diktion nimmt (Geißler 1925/34), und Verfasser des fachlich jämmerlichsten Beitrags im germanistisch-sprachwissenschaftlichen „Kriegseinsatz“ von 1941 (hierzu Hausmann 1998).

Als rhetorische Ressource betrachtet war Purismus ambivalent. Einerseits kritisierten die eingefleischten Puristen den NS-Sprachgebrauch, dessen Diktion den synkretischen Gesetzten erfolgreicher Propaganda folgte (die im 20. Jahrhundert den Gebrauch "wissenschaftlicher" Terminologie notwendig einschließt). Andererseits gab es nichts, was die Sprachpfleger dem Parteiapparat als Dienstleistung hätten anbieten können. So bietet sich, namentlich wenn man die Veröffentlichungen der "Muttersprache" von 1933 bis 1940 verfolgt, das Bild einer Gruppe, die vergeblich versucht, sich vorteilhaft ins Spiel zu bringen (eine Synopse solcher Beiträge gibt Kämper-Jensen 1993)23. Aus der (von Simon 1989 erforschten) Geschichte der Bemühungen um ein NS-Sprachamt wissen wir auch, daß der „Sprachverein“ es keiner NS-Dienststelle recht machen konnte, was aber überwiegend daran lag, daß Institutionen wie die Deutsche Akademie und später das Abnenerbe sich zutrauten, effizientere und modernere Sprachpolitik zu betreiben.

\section{Exemplarisches über die fachliche Einstellung zu „Wissenschaft" und „Wissenschaftlichkeit“}

Für die historische Wissenschaftsforschung gibt es kaum ein gravierenderes Problem als das der "Wissenschaft" selbst. Fortwährend verwandeln fachliche und außerfachliche Begebenheiten in ihrer Gesamtheit die "Wissenschaft" von gestern in den Aberglauben (günstigenfalls in das "Vorurteil“) von heute. Was den Nationalsozialismus betrifft, so hat die moralische Täter-Opfer-Optik den historischen Blick für Fachliches lange getrübt. Schuld, Verwicklungen und politische Allianzen waren (ganz zu Recht, wie ich finde) zuerst im Blick, aber wohl auch darum, weil das eigene Bild von "Wissenschaft ${ }^{\text {“ }}$ reingehalten und abgegrenzt werden sollte. Wer so schaut, der sieht nicht, daß sein Fach im Nationalsozialismus vielleicht nach ganz ähnlichen Resonanzgesetzen funktioniert hat wie heute. Die Vorstellung, es ließe sich im historischen Rückblick ein Kern unproblematischer "Wissenschaft" ausmachen und von politischen, ideologischen und sonstigen kontingenten „Zutaten“ reinlich trennen, ist naiv. Stattdessen sollte zur Fachgeschichte die Rekonstruktion der Auffassung gehören, die ihre jeweiligen Träger von "Wissenschaft" ausgebildet hatten. Und natürlich auch der, welche der Betrachter aus seiner (heutigen) Position für selbstverständlich zu halten geneigt ist!

${ }^{23}$ Dagegen ist die Fremdwort- und Fachwortanalyse des modernen "Sprachkämpfers" Schmidt-Rohr (vgl. 1933:415-423) äußerst differenziert und präzise. Gegenüber den heute am Markt erfolgreichen Lehren z.B. Uwe Pörksens würde ich sie jederzeit vorziehen. 
Nach dem soziologischen Lehrbuchwissen vollzieht sich die "Ausdifferenzierung" und kommunikative Autonomisierung der Disziplinen seit der Aufklärung. Regelmäßig stößt man auch auf Max Weber und dessen Analyse des „reinen“ wissenschaftlichen Ethos. Leider liest selten jemand weiter bis zur Weberschen Idealtypenlehre, wo dann steht, daß alle reinen Typen methodologische Konstrukte (und approximative Leitbilder!) sind und in der Wirklichkeit nicht unvermischt vorkommen.

In der Sprachwissenschaft kommt hinzu, worauf Utz Maas (1988) hingewesen hat, daß der forcierte und verspätete Anschluß der Deutschen an den „internationalen Strukturalismus “ in den 60er Jahren es leicht machte, die bis dahin tradierten fachlichen Bestände dadurch rasch zu entwerten, daß man sie pauschal als NS-infiziert recodierte (oder doch wenigstens als "vor-strukturalistisch" und vormodern).

Ich gebe exemplarisch einige Notizen zum Wissenschaftsverständnis von Georg Schmidt-Rohr, die mir zwar zugespitzt, aber doch darum typisch für die sprachwissenschaftliche Zunft scheinen, weil der Autor zwischen dem enragierten und politisierten Frontkämpfer- und Jugendbewegungsnationalismus und dem akademisch gedämpften völkischen Nützlichkeitsdenken seiner Universitätskollegen eine prekäre Mitte hält. Sein Hauptwerk $(1932,1933)$ wimmelt von bedauernden Bemerkungen darüber, daß er den gesamten wissenschaftlichen Apparat, die Fußnoten, die Auseinandersetzung mit der Literatur und den Fachkollegen aus Gründen der allgemeinen Verständlichkeit und der Breitenwirkung weggelassen habe.

In der 2. Auflage von 1933 sah er dann Anlaß, sich mit diversen Anwürfen auseinanderzusetzen, sein Werk sei „unwissenschaftlich“, und an diesen Einwürfen läßt sich rekonstruieren, welche Vorstellungen von „Wissenschaft“ im Jahre 1933 durch sprachwissenschaftliche Köpfe zirkulierten. Das Wissenschaftsethos dürfte ein zentraler Posten in der mentalen Ökonomie der deutschen Bildungsbürger (und in Sonderheit ihrer „Mandarine“) gewesen sein.

Von "gewissen Kreisen der Hochschulwissenschaft" sei sein Buch damit abgetan worden, daß es nicht im strengen Sinne „wissenschaftlich“ sei. Drei Lesarten dieses Vorwurfs unterscheidet Schmidt-Rohr:

(a) Kein einzelner könne den kompletten Katalog der Themen überschauen oder gar beherrschen, für welche die Sprache angeblich entscheidend sei: Erkenntnistheorie, Kulturphilosophie, Soziologie, Sprachphilosophie, vergleichende Sprachwissenschaft, Völkerpsychologie, Rassenkunde, Geschichte, Staatslehre, Ästhetik. Kurz: die umfassende „Zuständigkeitserklärung“ der Sprachforschung für alle kulturellen Hochwertsphären - kraft der Tatsache, daß der Zugang zu ihnen nur sprachlich möglich sei - gilt für bedenklich. Nicht zuletzt, weil damit alle anderen Geisteswissenschaften in die zweite Reihe verwiesen sind!

(b) Der zweite Anwurf lautet, es sei „unwissenschaftlich“, sich zur Entscheidung fachlicher Angelegenheiten an ein außerfachlich-allgemeines Publikum zu wenden (wie es Schmidt-Rohr ausdrücklich tut). Hier geht es offenbar darum, den sozial exklusiven Charakter der Wissenschaft zu wahren. 
(c) Drittens schließlich wird geäußert, es sei „unwissenschaftlich“, mit einem „gebundenen Standpunkt“ zu arbeiten, und zwar - hier muß ich zitieren - „nämlich dem, daß ich das gesunde Leben meines Volkes schützen wolle. Solche Zielsetzung sei von vornherein unwissenschaftlich; echte Wissenschaft sei wertfrei, zweckfrei, voraussetzungslos." (Schmidt-Rohr 1933: XI).

Natürlich beginnt Schmidt-Rohr mit der Bemerkung, daß sein Gewissen ihm alle diese Vorwürfe auch selbst schon gemacht habe, er sie aber in „Kämpfen um meine Verantwortung " niedergerungen habe (1933: XI). Woraus erhellt, daß er die praktische Verantwortung für das eigene „Volkstum“ wertmäßig über diese Einwände stellt. $\mathrm{Zu}$ (a) heißt es, natürlich könne keiner alles auf diesen Gebieten durcharbeiten, aber darum auf den Versuch der wissenschaftlichen Durchdringung des Sprache-Volk-Komplexes zu verzichten, das sei erst recht unwissenschaftlich. Zu (b) folgt eine der beim Publikum immer beliebten Schmähreden auf den exklusiven Jargon der Wissenschaft.

$\mathrm{Zu}$ (c) wird - das liegt nahe - die „reine“ Wissenschaft als volksfremder „Intellekt" und gleichgültige Verantwortungslosigkeit gegenüber dem Volksleben umgedeutet. Der (namentlich schriftinduzierte) Intellektualismus reduziere die wertmäßigen Verpflichtungsgehalte der Sprache und trage so zum Niedergang des

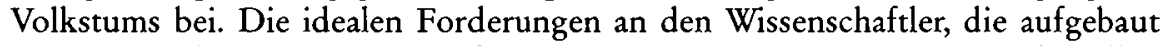
werden, reichen bis zum sacrificium intellectus um der Gemeinschaft willen (1933:377). Von den „Besserwissenden“ wird gefordert, daß sie zwar nicht lügen, aber schweigen können müssen. In einer Figur, die aus dem Kleistschen Marionettentheater stammen könnte, predigt Schmidt-Rohr, der dumpf vegetative Konsens einfach bodenständiger Volksgemeinschaften sei, da er naturwüchsig nicht mehr funktioniere, nunmehr mit modernen Mitteln bewußt zu leiten (1933: 381) ${ }^{24}$. Schmidt-Rohr beschwört das Gemeinschaftsapriori der Erkenntnis und die Herrschaftserfahrung der Kirchen. Man könnte ihn auch einen völkischen Gramsci nennen. „Der Wissenschaftler, der Gelehrte hat eine priesterliche Verantwortung gegenüber der Welt, in die er hineinspricht" (1933: XIII). Und, gewissermaßen als Sahnehäubchen auf der ganzen Argumentation, bietet Schmidt-Rohr am Ende ein Bekenntnis, das ihn angreifbar macht, weil es auch den von ihm in Anspruch genommenen Wert der Wissenschaft relativiert ${ }^{25}$. All denen, die sich seinen „unbequemen Gedankengängen“ mit der Ausflucht zu entziehen suchen, sie seien unwissenschaftlich, sagt er noch zuletzt:

${ }^{24}$ Damit sind wir bei einer Denkfigur, die in der späteren Soziologie Arnold Gehlens zunächst als oberste Führungssysteme, idées directrices, und später als "Institutionen“ Karriere machen sollte. Es wäre natürlich ein Leichtes, mit Zitaten zu belegen, daß diese Wissenschaftsauffassung höchst verbreitet war. Stroh (1933:1) beginnt bekenntnishaft, sein Buch sei vom "deutschen Standpunkt" aus geschrieben, der Psychologe Erich Jaensch spricht von den „Forderungen des gelebten Lebens" usw. All das ist eigentlich nicht mehr als eine (durch völkische Resonanz geschärfte) Spielart des philosophischen Pragmatismus, der auch anderwärts gedieh und gedeiht.

${ }^{25}$ Mit dem Vorwurf des Relativismus und Liberalismus fällt dann auch Glässer (1939) über ihn her. 
Ich glaube, daß es aus meiner Sprachphilosophie deutlich wird, daß es sich nicht beweisen läßt, was wissenschaftlich ist. Es läßt sich nur bekennen, was man für wissenschaftlich hält. Und dieses Bekenntnis wird je danach ausfallen, was für ein Mensch einer ist. (1933: XIII)

Das ist ein wahrhaft Mauthnerscher Satz. Da ist der Dauerkonflikt des TrotzigJugendbewegten mit den Parteistellen, zu denen er sich aus Pfadfinderehre bei aller Kritik immer loyal verhält, zum Greifen präsent. Die geistesgeschichtliche Pointe dabei ist freilich, daß die aufklärerische Sprach- und Erkenntnisskepsis Mauthners, gerade weil sie schwer zu widerlegen ist, den Weg mit freigemacht hat für völkische Aufladungen von Sprache und Wissenschaft selbst - nicht nur analytisch, sondern eben auch programmatisch.

Vergleicht man Schmidt-Rohrs Auslassungen zur "Wissenschaft" mit denen seiner stärker "akademisch" geprägten Kollegen, so findet man zwar durchaus einen anderen Ton, aber durchweg ähnliche Engführungen zwischen „Wissenschaft" und nationalem Interesse. Es scheint fast, als seien "Deutschtum" und Wissenschaft so nachhaltig miteinander identifiziert, daß gar nicht unwissenschaftlich sein kann, was dem deutschen Volke nützt. Nur so ist es zu erklären, $\mathrm{daß}$ z.B. in ein und demselben Text (Güntert 1932) die Pragmatisierung von Forschung und Erkenntnis wortreich als Entartung beklagt - und im Hinblick auf die deutschen Volksziele betrieben wird:

Die Aufsätze ruhen durchaus auf wissenschaftlichem, und zwar sprach- und kulturgeschichtlichem Grund und enthalten sich eines jeden Eingehens auf engere politische Parteifragen, ihr einziges Ziel ist Klarheit und Wahrheit, ihr einziger Sinn: dem Deutschtum zu seinem Recht zu verhelfen. (Güntert 1932: Vorwort)

Bereits die schiere Allgegenwart des Themas in den untersuchten sprachwissenschaftlichen Schriften ist Beleg für den Gärungsprozeß der Wissenschafts- und Erkenntnisbegriffe im fraglichen Zeitraum (vgl. die klassische Untersuchung von Ringer 1983 sowie Oexle 2000). Und wenn dann ein Autor aus der zweiten Reihe im Jahre 1936 krude resümiert:

Dadurch daß die Wissenschaft der neueren Sprachen sich in diesen Zusammenhang stellt [den der NS-Erziehungsidee; CK], büßt sie nichts von ihrer wissenschaftlichen Strenge ein. (Kißling 1936:339)

dann ist das zwar kaum communis opinio, aber gewiß das logische Ende einer national bzw. völkisch perspektivierten Wissens- und Erkenntnisauffassung. Zu dem expansiven System von Versprechungen, welches (vgl. Ehlich 1998) in das Amalgam der NS-Ideologie einschießt, gehört auch die Wiederherstellung Deutschlands als einer wissenschaftlichen Großmacht.

\section{Methodologische Schlußfolgerungen}

Zuerst gilt es, den Spielraum der zulässigen und resonanzfähigen Begriffe, Figuren und Geschichten auszuloten. Dazu ist es erforderlich, Vergleichbarkeit der Texte herzustellen und zu sichern. Ich habe die fachprogrammatischen Texte des „Stand 
und Aufgabe der Sprachwissenschaft" - Typs von Mitte der 20er bis Mitte der 30er Jahre untersucht ${ }^{26}$. Die Hypothese war, daß dieser Texttyp in der Hauptsache dazu dient, das Fach in einer veränderten „Umwelt“ rhetorisch und semantisch neu zu positionieren.

Wo es um die Vermittlungen zwischen Fach- und Theoriegeschichte auf der einen, Sozial- und Strukturgeschichte auf der anderen Seite geht, da interessieren semantische Bestände, Deutungsmuster, Argumentationen und „Geschichten“, die das Fach in seiner sozial-kommunikativen Umwelt resonanzfähig machen. Da die geisteswissenschaftlichen Fächer traditionell einen beträchtlichen Teil ihrer „Fachausdrücke“ aus der allgemeinen Bildungssprache und aus der philosophischen Tradition beziehen, ist das Studium der semantischen Resonanz zwischen Fach und Gesellschaft im Felde der Terminologie noch am einfachsten ${ }^{27}$. Ich bin der These nachgegangen, der semantische Komplex „Ganzheit“ (nebst den dazugehörigen „Feldnachbarn“ Struktur, System, Gestalt etc.) diene einerseits der außerfachlichen Resonanz, andererseits der innerfachlichen Vergesellschaftung über (ansonsten weitreichende) Theoriedifferenzen hinweg28. Dem ist hinzuzufügen, daß der Ganzheitsgedanke gleichzeitig auch als Brücke zwischen den geisteswissenschaftlichen Fächern zu dienen im Stande war, als eine Art Interdisziplinaritätssurrogat, als Suggestion, alle Vertreter von Ganzheitsgedanken zögen an einem (nationalen) Strang ${ }^{29}$. Auch das ist, ich wiederhole, eigentlich nichts beson-

26 Nicht eigens berücksichtigt habe ich in diesem Text den in jeder Hinsicht gewichtigen programmatischen Beitrag, den Leo Weisgerber 1933/34 in zwei Teilen in "Wörter und Sachen" publiziert hat, also unmittelbar nachdem er in deren Schriftleitung eingetreten war. Dieser Text bleibt zwar im oben abgesteckten Rahmen, skizziert aber ein weitreichendes und modernes sprachsoziologisches und varietätenlinguistisches Programm, das einer eingehenden Analyse bedürfte.

27 "Sofern wissenschaftliche Begriffe auch außerhalb der Wissenschaft verwendet werden, sind sie zum Einfallstor für externe Einflüsse einer besonderen Art prädestiniert; solche nämlich, deren Externalität von den Wissenschaftlern selbst schwer zu erkennen ist, weil sie ihnen als Teil ihres wissenschaftlichen Vokabulars entgegentreten. Ähnliches gilt von solchen Theorien und Erklärungsmustern, die sowohl alltäglich als auch wissenschaftlich verwendet werden. Es ist eine wichtige Aufgabe der Geistesgeschichte, das „Doppelleben“ von Ideen und seine Konsequenzen für die Wissenschaft zu erfassen“ (Scheerer 1991:112).

${ }^{28}$ In der Psychologie hat sich der „Ganzheitsgedanke" gar eine eigene deutsche Tradition erfunden, zu deren Stationen die Mystik und der deutsche Idealismus gerechnet wurden. Führend war hier Felix Krueger, der Wortführer der Leipziger Gestaltschule (vgl. Scheerer 1991). Ähnlich wie in der Psychologie konnte auch in der Sprachwissenschaft der Ganzheitsgedanke sogar die ansonsten unversöhnlichen Gegensätze zwischen "geisteswissenschaftlichen " und "naturwissenschaftlichen" Theorien überbrücken. Wobei eine deutliche Mehrheit der akademischen Zunftvertreter "naturwissenschaftliche " Orientierungen in der Sprachwissenschaft deutlich ablehnt und dem „überwundenen" 19. Jahrhundert zurechnet. Einen ernsthaften Brückenschlag zwischen beiden Perspektiven gibt es nur bei Bühler und wenigen anderen Psychologen.

${ }^{29}$ Zur Plausibilisierung dieses Gedankens möge der Hinweis auf die mehrbändige Festschrift zum 60. Geburtstag Felix Krugers dienen, die unter dem Titel „Ganzheit und Struktur" in den "Neuen psychologischen Studien" 1934 erschienen ist. Hier gibt es kaum einen Titel, in dem Ganzbeit, Struktur nicht vorkämen. Zu den Beiträgern gehören: Erich Jaensch, 
deres, denn sichtlich funktionieren die Leitbegriffe der Gegenwart ganz ähnlich. Auch „Struktur", „System“, „Funktion" tragen neben der Ganzheitssuggestion auch den (immer plausibilisierend wirkenden) Bezug zu anderen Disziplinen und auch zur außerfachlichen Kommunikation. $\mathrm{Daß}$ es inzwischen eine „ökologische", eine "natürliche " Linguistik, Psychologie etc. gibt, versteht sich von selbst, aber eben nicht aus binnenfachlichen Beweggründen, sondern aus solchen der (außerfachlichen) Resonanzfähigkeit.

Zu der spezifisch deutschen Art, auch sprachwissenschaftlich ,aufs Ganze zu gehen, läßt sich mit den Methoden der historischen Semantik und Diskursforschung eigentlich nur ausmachen, daß

a. die „deutschen“ Varianten des Ganzheitsdiskurses emphatisch, wertbetont und völkisch-national aufgeladen waren; die Zuständigkeit für das „Ganze“, für Synthese und Zusammenschau, galt den Akteuren der Zeit als Signum „deutscher" Wissenschaft;

b. die allgemeine Inklusionskrise der Weimarer Republik den Ganzheitsdiskurs „Zwangslos ${ }^{\text {“ }}$ an die Volksgemeinschaftsidee heranführte, ein „Zusammenrücken“ von fachlichem und völkischem Diskurs begünstigte;

c. der national-emphatische Ganzheitsdiskurs gleichzeitig und uno actu die gedemütigte Nation und die gedemütigte (ehedem weltweit führende) Disziplin zu kurieren versprach.

Insgesamt funktionieren Rhetorik und Semantik des "Ganzen" wie eine Rutschbahn, wie eine schiefe Ebene, die den fließenden Übergang zwischen dem sprachlichen Zeichensystem im engen Sinne und den kontingenten, aber mit Sprache "verbundenen" Sphären der gesellschaftlichen Kommunikation fallweise glättet. Indem Sprache am „Ganzen“ des gesellschaftlichen Lebens privilegiert teilhat, begründet sie symbolisch den Anspruch auf die Schlüsselposition der Sprachwissenschaft.

Von den überkommenen Weisen, die Geschichte der Sprachwissenschaft im Nationalsozialismus zu erzählen, ist keine frei von Paradoxien. Die geistes- und motivgeschichtliche (Römer 1985, Ahlzweig 1994) verfährt nolens volens immer ein wenig nach dem Muster der germanomanen Etymologien Jost Triers: Weil Ausdrücke wie „Rasse“, „Arier“, „Volk“ etc. für uns Nachgeborene unweigerlich mit den Konnotationen der NS-Praxis aufgeladen sind, scheinen sie „schon immer" auf diese hin entworfen oder aber "mißbraucht". Der höchst veränderliche rhetorische Ressourcen-Charakter der semantischen Motive und Bestände fällt dabei unter den Tisch $^{30}$. Den Rest besorgt die historiographische Gewohnheit, möglichst kohärente

Theodor Litt, Eduard Spranger, Hans Freyer, Max Wundt, Arnold Geblen, was den „interdisziplinären" Charakter dieser Veranstaltung zur Genüge zeigt.

30 Bausinger (1965:127) schreibt zu den Suggestionen geistesgeschichtlicher Kontinuität: „Zunächst fragt es sich ganz allgemein, ob das bemühte Aufsuchen geschichtlicher Vorstufen nicht häufig eine - freilich besonders subtile - Form des Ausweichens ist; so eindringlich eine Ouvertüre sein mag, das Spiel beginnt doch erst, wenn der Vorhang aufgeht." Das gewählte Sprachbild ist gewiß kritikwürdig, wahr bleibt indessen, was Hannah Arendt wiederholt vorgebracht hat: daß die Versuche der "geistesgeschichtlichen“ Ableitung der NS-Ideologie 
"Geschichten“ zu erzählen. Der zweite Stolperstein der Doxographie liegt, semiotisch gesprochen, in der naiven Interpretation der Quellen als „Kundgabe“ oder Bekenntnis, wo doch offenkundig das Ziel der rhetorischen Reproduktion des Faches unter den obwaltenden Bedingungen die Feder geführt hat (und folglich eine Deutung unter dem Organon-Aspekt der Rezipientensteuerung angemessener wäre als eine Deutung unter dem Aspekt des „Ausdrucks“!) ${ }^{31}$.

Die „Kriminalgeschichte“ der NS-Sprachwissenschaft, deren Meister ohne Zweifel Gerd Simon ist, zeigt das fachliche Tun der Protagonisten im Lichte ihrer Verwicklungen mit dem NS-Macht- und Gewaltapparat, die aber durchaus nicht zwingend mit dem fachlichen Wert in der einen oder in der anderen Richtung korrelieren müssen. Vielleicht sind diese Paradoxien Grund genug, es einmal mit den Mitteln der historischen Diskurs- und Begriffsforschung zu versuchen.

\section{Schluß: „Sprachgeschichte und Nationenkunde“; Eugen Lerch und die untere Anpassungslinie}

Ich komme zum Schluß - und damit zurück auf den Text von 1934, aus dem das Zitat zum Auftakt genommen war. Er heißt „Die neue Sprachwissenschaft. Sprachgeschichte und Nationenkunde “ und stammt von Eugen Lerch, der wenig später aus seiner Romanistik-Professur in Münster entlassen wurde ${ }^{32}$. Der fragli-

die Pointe der absoluten Prinzipienlosigkeit in der NS-Herrschaftstechnik verfehlen. Aufgelesen und eingebaut in den gemischten Chor des Nationalsozialismus wurden alle Stimmen, in denen Alternativen mit Aussicht auf Resonanz hätten artikuliert werden können. Mit dem Ergebnis, daß der Widerstand keine Sprachformen vorfand, die nicht selbst schon auf den Nationalsozialismus verwiesen hätten (vgl. Maas 1984). Wer sich eingehender mit der politischen Kommunikation in Massendemokratien befaßt hat, der sollte mit dieser rhetorischen Technik der „feindlichen Übernahme" potentiell resonanzfähiger Themen und Motive durch den Machtapparat bestens vertraut sein.

31 Darauf hat Maas (1996:73) hingewiesen: „Eine Analyse der heute z. T. grotesk anmutenden rhetorischen Verrenkungen muß solche zeitgenössischen Horizonte herausarbeiten, bei denen die institutionelle Reproduktion obenan steht, opportunistisch die aussichtsreichsten Argumente genutzt werden, um den institutionellen Interessen zur Geltung zu verhelfen (ist es heute anders?)." Das trifft insbesondere auf den mit viel historiographischem Abscheu bedachten Text von Hermann (1937) zu, dessen offenkundiges Ziel es war, die Göttinger Indogermanistik und Slavistik zu erhalten und auszubauen. 1937 wurde Hermann in Göttingen emeritiert.

32 Für Lerchs Entlassung war offenbar (neben einer Münsteraner Intrige) der Umstand maßgeblich, daß er mit einer Jüdin zusammenlebte. Mit Klemperer, von Wartburg und Spitzer zählte Lerch zu der Fraktion innerhalb der Romanistik, die gleichzeitig moderne (und das heißt: durch Saussure inspirierte synchronische) Sprachwissenschaft betreiben und an deren Daten "Nationenkunde " betreiben wollte. Das Zitat echot im übrigen einen Satz aus Friedrich Schürrs (1922) „Sprachwissenschaft und Zeitgeist“, einem viel gelesenen Text. Da heißt es (S. 18) inmitten einer wahren Ganzheitsorgie: "Hand in Hand mit dem Wiedererwachen der Philosophie und der Metaphysik geht heute auch in der Wissenschaft der Zug aufs Ganze." Auch in "Sprachwissenschaft und Zeitgeist" trifft man bereits rassistische Ableitungsfiguren, so daß die spätere NS-Affinität Schürrs jedenfalls nicht verwundert. 
che Beitrag aus den „Neueren Sprachen“ zählt noch zu den programmatischen Selbstthematisierungstexten des Faches aus den späten 20er und frühen 30er Jahren. Vor deren Hintergrund (vgl. Schürr 1922, 1925, Ipsen 1930, Bühler 1933, Weisgerber 1929, 1933/34, Güntert 1929, 1932, Hermann 1931, Dempe 1934, Funke 1928, Otto 1934) steht er für die Bearbeitung der Ambivalenz zwischen konsequenter fachlicher Modernität und Anerkennung des zu leistenden fachlichen Beitrages zur "Nationwerdung“. Es ist nicht ganz irrelevant für die Fachgeschichte, daß sich diese „direkte Kopplung“ $z$ wischen Systemlinguistik ${ }^{33}$ und "Nationenkunde“ nicht auf den kanonischen Saussure berufen kann. „Der“34 lehnt diesen Kurzschluß ausdrücklich ab, wie ich an anderer Stelle gezeigt habe (Knobloch 2000). Es ist auch nicht ganz irrelevant, daß es im sprachtheoretischen Denken eine Fraktion gab, die jeglichen Anschluß der Sprachstruktur an die „Nationenkunde“ ablehnte. Zu nennen wäre hier der Anglist Otto Funke ${ }^{35}$ (Bern), der über die „Neuromantiker“ um Weisgerber spottete, die ständig die „innere Sprachform" mit dem Weltbild des Sprechers verwechseln. Zu nennen wäre weiterhin Franz Dornseiff, der in einer Rezension von Weisgerber (1933/34) über die weit verbreitete "sprachphysiognomische Überreizung" spottet (Dornseiff 1934). Zu nennen wäre auch der ab 1939 in München lehrende Slawist Erwin Koschmieder, von dem eine auch heute noch vollkommen überzeugende Widerlegung der sprachnationalistischen und sprachrelativistischen Axiomatik stammt. Außerdem natürlich Karl Bühler und auch ein heute weitgehend unbekannter Sprachtheoretiker wie Hellmuth Dempe, der 1934 in den "Indogermanischen Forschungen“ gegen Güntert in dieser Sache aufgetreten ist. Es ist freilich auch nicht ganz zufällig, daß die nicht-nationale Richtung Otto Funkes, die das Etikett „empirisch“ im Schilde führte und sich auf den junggrammatisch nüchternen Hermann Paul berief, bereits in den 20er Jahren relativ isoliert war ${ }^{36}$.

33 Verbunden mit dem Anspruch, so auch erst die „wahre“ Sprachgeschichte geben zu können, weil die ja ebenfalls als Abfolge von Systemzuständen beschrieben werden müsse - mit Hinweis auf die Arbeiten Jost Triers.

${ }^{34}$ Die Anführungszeichen sollen andeuten, daß der kanonische Saussure ein Kollektivsubjekt ist, an dem die Person, deren Name das Titelblatt des "Cours“ schmückt, möglicherweise nur einen geringen Anteil hat.

35 Otto Funke war der einzige Schüler und konsequente Anhänger Anton Martys in der Sprachtheorie. Marty, ein unbequemer, umständlicher und weitgehend im Kampf gegen seine Zeitgenossen (vor allem gegen Steinthal und Wundt) verzettelter Theoretiker, war Schüler Franz Brentanos, von dem bekanntlich viele Einflüsse auf die moderne Sprachtheorie und die moderne Psychologie ausgegangen sind. Auch Dempe und Bühler sind von der Brentanoschule beeinflußt. Funke lehrte in Bern, und er notiert in einer Fußnote zu einem (durch und durch strukturellen) Aufriß der englischen Grammatik, den er 1944 in den „Englischen Studien" veröffentlichen konnte (Funke 1944), daß er den dort präsentierten Stoff im Jahre 1942 an den Universitäten Bonn und Marburg vorgetragen habe. Weisgerber ist 1942 von Marburg nach Bonn berufen worden. Von ihm gibt es einen wirklich bösartigen Angriff auf Funke aus den späten 20er Jahren (Weisgerber 1929). Das noch als Randbemerkung zum „Mythos $1^{\text {“. }}$ 36 Lerch hat seine Aufsatzsammlung von 1930 freilich noch dem Andenken von Hermann Paul gewidmet! 
Nicht nur kann man Lerch zur „unteren“ Selbstanpassungslinie zählen, weil er das „Ganze“ mit dem synchronischen „System" und mit „Nationenkunde“ identifiziert. Er ist auch darin typisch, daß er sich in Sachen Anwendungsrelevanz und Nützlichkeit auf den deutschen „Volks“-Diskurs einerseits, auf die Lehrerausbildung andererseits bezieht. Sein Beitrag ist auch darum interessant, weil er eine explizite terminologische Strategie enthält. Ich zitiere abschließend den Kernsatz dieser fachlichen Spracbregelung. Nachdem Lerch zunächst mit den später international durchgesetzten Termini „System“ und „Struktur" beginnt, fährt er fort:

Statt der Ausdrücke „Struktur" und „System“ würde ich lieber die Ausdrücke „Gefüge" oder "Wesensgestalt" "gebrauchen. Die Sprache hat für mich (und für v. Wartburg) ein Gefüge, eine Wesensgestalt. (Lerch 1934:376)

Mit Hilfe der emphatischen Sinn- und Wertaufladung der „Wesen“-Semantik läßt sich die nationale Wesenskunde direkt an die strukturelle Grammatik anschließen. Und das war ja der Stoff, von welchem sich die (durch Lerch bereits kurz nach dem Ersten Weltkrieg ausgelöste) Debatte um den „Dauerfranzosen“ nährte (vgl. Jehle 1996:47-50, Hausmann 1998:331ff.). Neben dem Hang zum „Ganzen“ gehört die Anerkennung der national-kulturellen Eigenwerte der Sprachen in Struktur und Geschichte (und die Anerkennung der Sprachwissenschaft als „zuständig “ für diese Eigenwerte) zur rhetorisch-semantischen Minimalausstattung einer resonanzfähigen Sprachwissenschaft im Nationalsozialismus. Auch Lerch geht relativ weit in der nationalen Rhetorik. So zitiert er ständig von Wartburg als Autorität, vor allem aus dessen programmatischem Artikel „Über die Bildungswerte des sprachwissenschaftlichen Studiums“ (Wartburg 1934), der kollusionssemantisch nicht gerade zurückhaltend verfährt. Lerch schließt mit einem Zitat von Wartburgs, in dem dieser die „Wesensunterschiede“ zwischen dem Französischen und dem Italienischen resümiert:

Eine Gegenüberstellung der beiden in Geschichte und Gegenwart öffnet dem Betrachter die Augen dafür, was die Verschiedenheit des Schicksals, des Bodens, des Blutes für das Werden des menschlichen Geistes bedeutet, zugleich aber auch wie Erziehung einer ganzen Nation und gemeinsamer Kulturwille den Geist und durch den Geist dessen vornehmsten Ausdruck, die Sprache, zu gestalten und umzugestalten vermögen. (von Wartburg 1934, zit. nach Lerch 1934: 397)

Hier haben wir gewissermaßen in einer semantischen Nußschale die später beinahe kanonische Position der völkisch-sprachnationalistischen Richtung: Schicksal, Blut und Boden als entscheidend für das "Werden“ des Volksgeistes - und Sprache nebst Kulturwille als entscheidend für die der Erziehung zugängliche "geistige Gemeinschaft ${ }^{\text {“37 }}$. So konnte die akademische Intelligenz, die auf logische

37 Der Innsbrucker Sprachwissenschaftler Hermann Ammann (seit gemeinsamen Studienzeiten in Freiburg mit Hans F. K. Günther, dem „Rasse-Günther", gut befreundet) findet ebenfalls 1934 eine ganz ähnliche Formel: „Träger der Sprache ist die Sprachgemeinschaft, ist das Sprachvolk, dessen kulturelle Einheit auf der tieferliegenden Natureinheit des gemeinsamen Bluterbes ruht. Die Überlieferung der Sprache ist, soweit sie für den einzelnen Sprecher Muttersprache ist, an die Bande des Blutes geknüpft. Die Sprache lebt, solange sie dem Leben 
Folgerichtigkeit ihrer „Weltanschauung “ mehr Wert legte als die NS-Parteiprominenz, immerhin mitteilen, daß Juden, Liberale und Kommunisten nicht per se, d.h. per Sprache, zur Volksgemeinschaft gehören sollten.

\section{Literatur}

(Ablzweig 1994) Claus Ablzweig, Muttersprache - Vaterland. Die deutsche Nation und ihre Sprache (Opladen 1994).

(Ammann 1934) Hermann Ammann, Vom Sinn der Sprachgeschichte, in: Neue Jahrbücher für Wissenschaft und Jugendbildung 10 (1934) 530-537.

(Ash \& Geuter 1986) Mitchell G. Ash, Ulfried Geuter, Geschichte der deutschen Psychologie im 20. Jahrhundert (Opladen 1986).

(Bausinger 1965) Hermann Bausinger, Volksideologie und Volksforschung, in: Deutsches Geistesleben und Nationalsozialismus, hrsg. v. Andreas Flitner (Tübingen 1965) 125-143.

(Bernsmeier $1977 \mathrm{ff}$.) Helmut Bernsmeier, Der Allgemeine Deutsche Sprachverein. 3 Teile, in: Muttersprache 87 (1977) 369-395 (Teil 1), 90 (1980) 117-140 (Teil 2) u. 93 (1983) 35-58 (Teil 3).

(Boebm 1932) Max Hildebert Boebm, Das eigenständige Volk (Darmstadt 21965).

(Bübler 1931/32) Karl Bübler, Das Ganze der Sprachtheorie, ihr Aufbau und ihre Teile. Bericht über den 12. Kongreß der deutschen Gesellschaft für Psychologie in Hamburg (Jena 1931/32) 95-122.

(Bübler 1933) Karl Bübler, Die Axiomatik der Sprachwissenschaften, in: Kant-Studien 38, (1933) 19-90.

(Burkamp 1929) W. Burkamp, Die Struktur der Ganzheiten (Berlin 1929).

(Dempe 1934) Hellmuth Dempe, „Sprache und Volksgeist“. Grundsätzliche Betrachtungen im Anschluß an Hermann Günterts Sprachauffassung in seiner Schrift „Deutscher Geist“, in: Indogermanische Forschungen 52 (1934) 89-107.

(Dornseiff 1934) Franz Dornseiff, Sprache und Gesamtkultur, in: Geistige Arbeit 1, Nr. 12 (1934) 8-13.

(Dornseiff 1938) Franz Dornseiff, Das Problem des Bedeutungswandels, in: Zeitschrift für deutsche Philologie 63 (1938) 119-138.

(Ehlers 2000) Klaas-Hinrich Ehlers, Saussure-Lektüre in Weisgebers Habilitationsschrift, in: Interpretation und Re-Interpretation. Aus Anlaß des 100. Geburtstages von Johann Leo Weisgerber (1899-1985), hrsg. v. Klaus D. Dutz (Münster 2000) 51-66.

(Ehlich 1989) Konrad Ehlich, Sprache im Faschismus (Frankfurt a. M. 1989).

(Ehlich 1998) Konrad Eblich, ...LTI, LQI,... Von der Unschuld der Sprache und der Schuld der Sprechenden, in: Kämper, Schmidt (1998) 275-304.

(Funke 1927) Otto Funke, Studien zur Geschichte der Sprachphilosophie (Bern 1927).

(Ganzheit und Struktur 1934) Ganzheit und Struktur. Festschrift zum 60. Geburtstage Felix Kruegers, hrsg. v. Otto Klemm, Hans Volkelt, Karlfried Graf v. Dürckbeim-Montmartin (Neue Psychologische Studien 12, Heft 1-3,1934).

(Geuter 1984) Ulfried Geuter, Die Professionalisierung der deutschen Psychologie im Nationalsozialismus (Frankfurt a. M. 1984).

(Glässer 1939) Edgar Glässer, Einführung in die rassenkundliche Sprachforschung. Kritischhistorische Untersuchungen (Kulturgeschichtliche Bibliothek, NF, zweite Reihe, Band 1, Heidelberg 1939).

dient und den Gesetzen unterworfen ist, nach denen Leben sich erneuert." (Ammann 1934: 535). 
(Güntert 1929) Hermann Güntert, Zum heutigen Stand der Sprachforschung, in: Wörter und Sachen 12 (1929) 386-396.

(Güntert 1932) Hermann Güntert, Deutscher Geist. Drei Vorträge (Bühl, Baden 1932).

(Hartmann 1998) Silvia Hartmann, Fraktur oder Antiqua. Der Schriftstreit von 1881 bis 1941 (Frankfurt a.M., Berlin 1998).

(Harvolk 1990) Edgar Harvolk, Eichenzweig und Hakenkreuz. Die Deutsche Akademie in München (1924-1962) und ihre volkskundliche Sektion (München 1990).

(Hausmann 1998) Frank-Rutger Hausmann, „Deutsche Geisteswissenschaft“ im Zweiten Weltkrieg. Die „Aktion Ritterbusch“ (1940-1945) (Dresden 1998).

(Hellpach 1937) Willy Hellpach, Einzelheit und Ganzheit, in: Industrielle Psychotechnik 13,5 (1937) 129-139.

(Hellpach 1938) Willy Hellpach, Einführung in die Völkerpsychologie (Stuttgart 1938).

(Hermann 1931) Eduard Hermann, Der heutige Stand der Sprachwissenschaft, in: Zeitschrift für Deutschkunde (1931) 145-154.

(Hermann 1937) Eduard Hermann, Was hat die indogermanische Sprachwissenschaft dem Nationalsozialismus zu bieten?, in: Göttingische Gelehrte Anzeigen 199, 2-3 (1937) 49-59.

(Hutton 1999) Christopher M. Hutton, Linguistics and the Third Reich: Race, Mother Tongue Fascism and the Science of Language (London 1999).

(Ipsen 1930) Gunther Ipsen, Sprachphilosophie der Gegenwart (Philosophische Forschungsberichte Nr. 6, Berlin 1930).

(Jeble 1996) Peter Jeble, Werner Krauss und die Romanistik im NS-Staat (Berlin 1996).

(Kämper-Jensen 1993) Heidrun Kämper-Jensen, Spracharbeit im Dienst des NS-Staats, in: Zeitschrift für germanistische Linguistik 21 (1993) 150-183.

(Kißling 1936) Helmut Kißling, Sprache und Volksgeist, in: Die neueren Sprachen 44 (1936) 339-356.

(Klingemann 1996) Carsten Klingemann, Soziologie im Dritten Reich (Baden-Baden 1996).

(Kloss 1935) Heinz Kloss, Fremdsprachige Einwanderung in das französische Sprachgebiet Frankreichs vor dem Weltkriege (Berlin 1935).

(Kloss 1937) Heinz Kloss, Um die Einigung des Deutschamerikanertums. Geschichte einer unvollendeten Volksgruppe (Berlin 1937).

(Kloss 1944) Heinz Kloss, Statistik, Presse und Organisationen des Judentums in den Vereinigten Staaten und Kanada (Stuttgart, Selbstverlag der Publikationsstelle Stuttgart-Hamburg [Nur für den Dienstgebrauch] 1944).

(Knobloch 1988) Clemens Knobloch, Geschichte der psychologischen Sprachauffassung in Deutschland von 1850 bis 1920 (Tübingen 1988).

(Knobloch 2000) Clemens Knobloch, Begriffspolitik und Wissenschaftsrhetorik bei Leo Weisgerber, in: Interpretation und Re-Interpretation. Aus Anlaß des 100. Geburtstages von Johann Leo Weisgerber (1899-1985), hrsg. v. Klaus D. Dutz (Münster 2000) 145-174.

(Knobloch i. V.) Clemens Knobloch, Über die Sprache der völkischen Sprachwissenschaft vor und nach 1933: Georg Schmidt-Rohr (i. V.).

(Krueger 1932) Felix Krueger, Das Problem der Ganzheit, in: Blätter für deutsche Philosophie 6 (1932) 111-139.

(Lerch 1930) Eugen Lerch, Hauptprobleme der französischen Sprache (Braunschweig 1930).

(Lerch 1934) Eugen Lerch, Die neue Sprachwissenschaft. Sprachgeschichte und Nationenkunde, in: Die Neueren Sprachen 9 (1934) 375-397.

(Lerchenmüller 1997) Joachim Lerchenmüller, Keltischer Sprengstoff. Eine wissenschaftsgeschichtliche Studie über die Keltologie von 1900 bis 1945 (Tübingen 1997).

(Maas 1984) Utz Maas, Als der Geist der Gemeinschaft eine Sprache fand - Sprache im Nationalsozialismus (Opladen 1984).

(Maas 1985) Utz Maas, Konnotation. Politische Sprachwissenschaft, hrsg. v. Franz Januschek (Opladen 1985) 71-96.

(Maas 1988) Utz Maas, Die Entwicklung der deutschsprachigen Sprachwissenschaft von 
1900 bis 1950, zwischen Professionalisierung und Politisierung, in: Zeitschrift für germanistische Linguistik 16 (1988) 253-290.

(Maas 1988a) Utz Maas, Sprachwissenschaft und Nationalsozialismus, in: Semiotische Berichte 12 (1988) 249-264.

(Maas 1988b) Utz Maas, Probleme und Traditionen der Diskursanalyse, in: ZPSK 41 (1988) 717-729.

(Maas 1989) Utz Maas, Sprachpolitik und politische Sprachwissenschaft (Frankfurt a.M. 1989).

(Maas 1996) Utz Maas, Verfolgung und Auswanderung deutschsprachiger Sprachforscher 1933-1945, Bd. 1 (Osnabrück 1996).

(Oexle 2000) Otto Gerhard Oexle, Zusammenarbeit mit Baal. Über die Mentalität deutscher Geisteswissenschaftler 1933 - und nach 1945, in: Historische Anthropologie 8,1 (2000) 127.

(Otto 1934) Ermst Otto, Grundfragen der Linguistik, in: Indogermanische Forschungen (1934) 177-195.

(Petzold 1978) Joachim Petzold, Wegbereiter des deutschen Faschismus. Die Jungkonservativen in der Weimarer Republik (Köln 1978).

(Ringer 1983) Fritz $K$ Ringer, Die Gelehrten. Der Niedergang der deutschen Mandarine 1890-1933 (Stuttgart 1983).

(Römer 1985) Ruth Römer, Sprachwissenschaft und Rassenideologie in Deutschland (München 1985).

(Sauer 1985) Christoph Sauer, NS-Sprachpolitik in der Besatzungssituation. Januschek (1985) 271-306.

(Sauer 1989) Christoph Sauer, Nazi-Deutsch für Niederländer. Das Konzept der NSSprachpolitik in der Deutschen Zeitung in den Niederlanden 1940-1945. Eblich (1989) 237-288.

(Sauer 1995) Christoph Sauer, Sprachwissenschaft und NS-Faschismus. Lehren aus der sprachwissenschaftlichen Erforschung des Sprachgebrauchs deutscher Nationalsozialisten und Propagandisten für den mittel- und osteuropäischen Umbruch?, in: Klaus Steinke (Hrsg.), Die Sprache der Diktaturen und Diktatoren (Heidelberg 1995) 9-96.

(Scheerer 1991) Eckart Scheerer, Vom Nutzen der Geistesgeschichte für die Psychologiegeschichte, in: Theorien und Methoden psychologiegeschichtlicher Forschung, hrsg. v. Helmut E. Lück, Rudolf Miller (Göttingen 1991).

(Schmidt-Robr 1917) Georg Schmidt-Robr, Unsere Muttersprache als Waffe und Werkzeug des deutschen Gedankens (Jena 1917).

(Schmidt-Robr 1932) Georg Schmidt-Robr, Die Sprache als Bildnerin der Völker (Jena 1932). (2. Aufl. unter dem Titel: Mutter Sprache. Vom Amt der Sprache bei der Volkswerdung, Jena 1933).

(Schmidt-Rohr 1933) Georg Schmidt-Robr, Volksfeindliche Sprachphilosophie?, in: Deutsche Kulturwacht. Blätter des Kampfbundes für deutsche Kultur 27 (1933) 14-15.

(Schmidt-Rohr 1934) Georg Schmidt-Rohr, Rasse und Sprache. Eine Entgegnung, in: Zeitschrift für Deutschkunde 48 (1934) 318-323.

(Schmidt-Robr 1935) Georg Schmidt-Rohr, Houston Stewart Chamberlain über die deutsche Sprache, in: Muttersprache 50 (1935) 301-305.

(Schmidt-Robr 1939a) Georg Schmidt-Robr, Rasse und Sprache, in: Rasse. Monatsschrift für den nordischen Gedanken 6,3 (1939) 161-168.

(Schmidt-Robr 1939b) Georg Schmidt-Rohr, Die zweite Ebene der Volkserhaltung, in: Rasse. Monatsschrift für den nordischen Gedanken, 6,4 (1939) 81-89. [1939a und 1939b nachgedruckt in Heft 7 und 8 der Muttersprache 54/1939]

(Schmidt-Rohr 1940a) Georg Schmidt-Robr, Vom Sprachgedanken in Kriegszeiten, in: Muttersprache 55 (1940) 145-147.

(Schmidt-Rohr 1940b) Georg Schmidt-Rohr, Rassebewußtsein - Rassenbewußtsein?, in: Muttersprache 55 (1940) 33-35. 
(Schürr 1922) Friedrich Schürr, Sprachwissenschaft und Zeitgeist. Eine sprachphilosophische Studie (Marburg 1922).

(Schürr 1923) Friedrich Schürr, Das Wesen der Sprache und der Sinn der Sprachwissenschaft, in: Deutsche Vierteljahresschrift für Literaturwissenschaft und Geistesgeschichte 1 (1923) $469-490$.

(Simon 1985) Gerd Simon, Die sprachsoziologische Abteilung der SS. Sprachtheorie, Pragmatik, Interdisziplinäres (Akten des 19. Linguistischen Kolloquiums Vechta 1984, Tübingen 1985) 375-396.

(Simon 1986) Gerd Simon, Der Wandervogel als „Volk im Kleinen“ und Volk als Sprachgemeinschaft beim frühen Georg Schmidt (-Rohr), in: Sprachwissenschaft und Volkskunde. Perspektiven einer kulturanalytischen Sprachbetrachtung, hrsg. v. H. E. Brekle, U. Maas (Opladen 1986) 155-184.

(Simon 1986a) Gerd Simon, Wissenschaft und Wende 1933. Zum Verhältnis von Wissenschaft und Politik am Beispiel des Sprachwissenschaftlers Georg Schmidt-Rohr, in: Das Argument 158 (1986) 527-542.

(Simon 1989) Gerd Simon, Sprachpflege im „Dritten Reich“, in: Sprache im Faschismus, hrsg. v. Konrad Eblich (Frankfurt a.M. 1989) 58-87.

(Simon 1990) Gerd Simon, Die Bemühungen um Sprachämter und ähnliche Norminstanzen im Deutschland der letzten hundert Jahre, in: Sprachnorm und Sprachnormierung, hrsg. v. W. Settekorn (Wilhelmsfeld 1990) 69-84.

(Stempel 1978) Wolf-Dieter Stempel, Gestalt, Ganzheit, Struktur. Aus Vor- und Frühgeschichte des Strukturalismus in Deutschland (Göttingen 1978).

(Strob 1933) Fritz Strob, Der volkhafte Sprachbegriff (Halle a. S. 1933).

(Thierfelder 1938) Franz Thierfelder, Deutsch als Weltsprache (Berlin 1938).

(Thierfelder 1940) Franz Thierfelder, Englischer Kulturimperialismus (Schriften des Deutschen Instituts für außenpolitische Forschung Nr. 26, Berlin 1940).

(Thierfelder 1941) Franz Thierfelder, Sprachpolitik und Rundfunk (Berlin 1941).

(von Wartburg 1934) Walther von Wartburg, Über die Bildungswerte des sprachwissenschaftlichen Studiums, in: Neuphilologische Monatsschrift (1934).

(Weisgerber 1925) Leo Weisgerber, Sprache als gesellschaftliche Erkenntnisform (Ungedruckte Habilschrift, Phil. Fak. 1924, Bonn 1925).

(Weisgerber 1929) Leo Weisgerber, Muttersprache und Geistesbildung (Göttingen 1929).

(Weisgerber 1931) Leo Weisgerber, Artikel „Sprache“, in: Handwörterbuch der Soziologie, hrsg. von Alfred Vierkandt (Stuttgart 1931) 592-608.

(Weisgerber 1933/34) Leo Weisgerber Die Stellung der Sprache im Aufbau der Gesamtkultur (Teil 1: Wörter und Sachen 15. 5-96. Teil 2: Wörter und Sachen 16, 1933/34) 97-236.

(Weisgerber 1934) Leo Weisgerber, Sprachgemeinschaft und Volksgemeinschaft und die Bildungsaufgaben unserer Zeit, in: Zeitschrift für deutsche Bildung (1934) 289-303. 
